\title{
The influence of processed total non-forward and non-motile sperm count on the outcome of artificial insemination with the husband's semen
}

\author{
Lan Liu, Bingping Wu, Zhimin Li, Hua Lin \\ Reproductive Medical Center, The Affiliated Hospital (Group) of Putian University, Fujian, China \\ Contributions: (I) Conception and design: L Liu, B Wu; (II) Administrative support: B Wu; (III) Provision of study materials or patients: L Liu; (IV) \\ Collection and assembly of data: B Wu; (V) Data analysis and interpretation: Z Li, H Lin; (VI) Manuscript writing: All authors; (VII) Final approval \\ of manuscript: All authors. \\ Correspondence to: Bingping Wu. Reproductive Medical Center, The Affiliated Hospital (Group) of Putian University, Dongzhen East Road No.999, \\ Putian, China. Email: bingping.68@163.com.
}

Background: Artificial insemination with the husband's semen (AIH) is an economical and noninvasive
method of infertility treatment. However, AIH's pregnancy rate is much lower than in vitro fertilization (IVF)
as its multiple and complex uncertainty factors. Semen quality has been one of the main factors which affect
the pregnancy outcome of AIH.
Methods: The relevant parameters of $1,142 \mathrm{AIH}$ cycles were retrospectively studied, including the general
parameters and the semen quality parameters among clinical pregnancy, biochemical pregnancy, non-
pregnancy group, age, infertility duration, infertility type, body mass index (BMI), cycle count, morphology
in previously semen examination, and semen quality parameters on the day of AIH.
Results: The statistically significant difference was only found on processed total non-forward and non-
motile sperm count (N-TFMSC). The mean processed N-TFMSC in the biochemical pregnancy group
was $6.37 \pm 4.27$ million, significantly higher than the other two groups (vs. $4.40 \pm 3.15$ million or vs. $4.48 \pm$
3.60 million, P<0.05). The study was then divided into two groups according to processed N-TFMSC,
Group $1 \leq 5.0$ million, and Group $2>5.0$ million. A statistical increase in biochemical pregnancy rate was
observed when the processed N-TFMSC was $>5.0$ million $(2.72 \%$ s. $0.90 \%)$.

Conclusions: Processed N-TFMSC may be one of the independent factors on AIH's outcome; it should be given equal attention the same as processed total forward motile sperm count (TFMSC).

Keywords: Artificial insemination with husband's semen (AIH); total non-forward and non-motile sperm count
(N-TFMSC); biochemical pregnancy

Submitted Oct 13, 2020. Accepted for publication Dec 02, 2020.

doi: $10.21037 /$ tau-20-1385

View this article at: http://dx.doi.org/10.21037/tau-20-1385

\section{Introduction}

Artificial insemination with the husband's semen (AIH) is an economical and noninvasive method of infertility treatment; the optimized semen is delivered directly into the female uterine to achieve a pregnancy $(1,2)$. However, AIH's pregnancy rate is much lower than in vitro fertilization (IVF) as its multiple and complex uncertainty factors $(3,4)$. $\mathrm{AIH}$, as an assisted reproductive technology which is closer to the natural pregnancy process, is the first choice for the treatment of infertility such as semen abnormality, sexual dysfunction and female cervical factors. The pregnancy rate of AIH reported in the literature fluctuated from $8 \%$ to $22 \%$, which was affected by the age of the woman, infertility factors, ovulation promotion program, semen quality and other factors. Semen quality is one of the main factors which affect the pregnancy rate of AIH. More 
research concerns the processed total forward motile sperm count (TFMSC) as the critical parameter of semen quality $(5,6)$ because only forward motile sperm can reach to the oviduct and occur fertilization. Simultaneously, the nonforward and non-motile sperm is also delivered directly into the female uterine cavity, together with the forward motile sperm. Although they do not compete for fertilization, do they have any other adverse effects on AIH?

We retrospectively analyzed $1,142 \mathrm{AIH}$ cycles performed in our Reproductive Medical Center between January 2016 and December 2019. Series parameters are compared among clinical pregnancy, biochemical pregnancy, and non-pregnancy groups, including age, infertility duration, infertility type, body mass index (BMI), cycle count, morphology in previously semen examination, and semen quality parameters on the day of AIH. Here, TFMSC, total non-forward and non-motile sperm count(N-TFMSC) are defined as the relevant semen quality parameters.

We present the following article in accordance with the MDAR reporting checklist (available at http://dx.doi. org/10.21037/tau-20-1385).

\section{Methods}

\section{Study design}

The relevant parameters of 1,142 AIH cycles performed in our Reproductive Medical Center between January 2016 and December 2019 were retrospectively analyzed. The study was approved by The Affiliated Hospital (Group) of Putian University (NO.[2015]229). All infertile patients who conformed to the AIH indications were enrolled in the study and signed the informed consent. All procedures performed in this study involving human participants were in accordance with the Declaration of Helsinki (as revised in 2013). The general parameters (including age, infertility duration, infertility type, BMI, cycle count, morphology in previously semen examination) and the semen quality parameters (pre-processed TFMSC, preprocessed N-TFMSC, processed TFMSC, processed N-TFMSC) were compared among clinical pregnancy, biochemical pregnancy, and non-pregnancy group. The semen quality parameters (motility and density) were tested by a sperm quality analyzer (WLJY-9000, China), the semen morphology was analyzed with the modified Papanicolaou staining method. The study was then divided into two groups according to the processed N-TFMSC to explore the influence on the outcome of AIH, Group 1 $\leq 5.0$ million, and Group $2>5.0$ million of the processed N-TFMSC.

\section{Ovulation monitoring}

Females with regular menstrual cycles could adopt AIH in natural cycles. Follicular development was monitored by transvaginal ultrasound from day 10 of the cycle or onward according to the menstrual cycle length. When the dominant follicle diameter reached $14 \mathrm{~mm}$, the urine luteinizing hormone (LH) peak was monitored. AIH was performed about $24 \mathrm{~h}$ after the occurrence of the LH peak. The stimulated cycle was controlled primarily by letrozole (Jiangsu, Hengrui Pharmaceutics, Lianyungang, China) or gonadotropin (HMG, Lizhu Pharmaceutics, Zhuhai, China; FSH, Lizhu Pharmaceutics, Zhuhai, China). Transvaginal ultrasound monitored follicular development (GE, Voluson E6, USA) and the urine LH. An intramuscular injection of 10,000 IU HCG (Maanshan, Fengyuan Pharmaceutics, Maanshan, China) was administered when the dominant follicle diameter reached $18 \mathrm{~mm}$, or the LH peak appeared. AIH was performed about 24-48 h after the intramuscular injection.

\section{Semen collection and processing}

Semen collection and processing is done according to the guidelines of the World Health Organization (WHO) procedures. Masturbation was used to collect semen after abstinence for 2-7 days and placed in a $\mathrm{CO}_{2}$ incubator to liquefaction. After the semen was processed through the density gradient centrifugation or the upstream method, $0.5 \mathrm{~mL}$ of semen suspension was prepared and used for the artificial insemination within an hour. Routine analysis of semen was performed before or after processing. Here, we defined the TFMSC as the multiplication of density, activity ratio of $(a+b)$ grade and volume of the sperm, and defined the N-TFMSC as the multiplication of density ratio $(\mathrm{c}+\mathrm{d})$ grade and the volume of the sperm.

\section{Artificial insemination}

Patients were placed in a lithotomy position. After vaginal and cervical secretions were cleaned, $0.5 \mathrm{~mL}$ of semen suspension was slowly injected into the uterine cavity through a disposable intrauterine insemination tube (COOK, USA). The patients were instructed to keep in the dorsal decubitus for $30 \mathrm{~min}$ after the operation. 
Table 1 Profile of patients in the clinical pregnancy, biochemical pregnancy, and non-pregnancy group

\begin{tabular}{lccc}
\hline General parameters & Clinical pregnancy & Biochemical pregnancy & Non-pregnancy \\
\hline Cycles $(\mathrm{n})$ & 143 & 17 & 982 \\
Female age (year) & $29.95 \pm 3.53$ & $30.41 \pm 3.36$ & $30.38 \pm 3.89$ \\
Male age (year) & $31.53 \pm 4.14$ & $32.24 \pm 3.27$ & $31.91 \pm 4.60$ \\
Infertility duration (year) & $2.76 \pm 1.84$ & $3.00 \pm 2.15$ & $2.87 \pm 1.95$ \\
Primary infertility (\%) & $61.54(88 / 143)$ & $64.71(11 / 17)$ & $62.42(613 / 982)$ \\
Stimulated AlH cycle (\%) & $46.15(66 / 143)$ & $29.41(5 / 17)$ & $51.12(502 / 982)$ \\
Female BMl & $21.97 \pm 3.59$ & $23.45 \pm 3.57$ & $21.90 \pm 3.36$ \\
Cycle count & $1.90 \pm 1.03$ & $1.94 \pm 0.83$ & $1.85 \pm 0.89$ \\
Morphology (\%) & $4.40 \pm 2.56$ & $4.72 \pm 1.82$ & $4.27 \pm 2.12$ \\
\hline
\end{tabular}

Values are mean \pm SD; One-way ANOVA or non-parametric test was used in this analysis. AlH, artificial insemination with the husband's semen; BMI, body mass index.

\section{Diagnosis of pregnancy}

Blood $\beta$-hCG was determined in patients 14-16 days after AIH. B-ultrasound was performed in patients with positive $\beta$-hCG 35 days after AIH. Gestational sac and primitive heart tube pulsation are regarded as clinical pregnancy. Patients with positive $\beta$-hCG while no gestational sac are regarded as biochemical pregnancy.

\section{Statistical analysis}

Statistical analysis was performed with SPSS 19.0 software. The measurement data in multiple groups were compared with randomized analysis of variance, and the comparison among groups was performed with LSD test (One-way ANOVA). If the measurement data disobey the Gauss distribution, a non-parametric test is used to analyze. A comparison between the two groups was constructed with an independent sample $T$-test. The enumeration data in multiple groups were displayed as crosstabs and were compared with the $\chi^{2}$ test. Statistical significance was established at $\mathrm{P}<0.05$.

\section{Results}

\section{The general data on the outcome of AIH}

The study involved 1,142 AIH cycles; the total clinical pregnancy rate was $12.52 \%(143 / 1,142)$, the total biochemical pregnancy rate was $1.49 \%(17 / 1,142)$. The general data were divided into three groups according to the outcome of AIH. As shown in Table 1, there was no statistically significant difference in the general parameters among the three groups, including female age, male age, infertility duration, primary infertility, insemination cycle, female BMI, cycle count, and the morphology in previously semen examination (all $\mathrm{P}>0.05$ ).

\section{Semen quality parameters on the outcome of AIH}

Here, TFMSC and N-TFMSC before or after processing were determined to be the semen quality parameters. As shown in Table 2, the mean processed N-TFMSC in the biochemical pregnancy group was $6.37 \pm 4.27$ million, significantly higher than the other two groups (vs. $4.40 \pm 3.15$ million or $v s .4 .48 \pm 3.60$ million, $\mathrm{P}<0.05)$. There were no statistically significant differences in pre-processed TFMSC, pre-processed N-TFMSC, and processed TFMSC.

\section{The influence of processed N-TFMSC on the outcome of $A I H$}

The study was divided into two groups according to processed N-TFMSC, Group 1: $\leq 5.0$ million (786 AIH cycles), and Group 2: >5.0 million (356 AIH cycles). The significant difference we observed between them tend to be more male parameters, including male age $(31.68 \pm 4.46 v s$. $32.27 \pm 4.66)$, morphology in previously semen examination $(4.08 \pm 2.01$ vs. $4.74 \pm 2.43)$, and all the semen quality parameters on the day of AIH (in Table 3). The enumeration 
Table 2 Semen quality parameters of patients on the day of AIH in the clinical pregnancy, biochemical pregnancy, and non-pregnancy

\begin{tabular}{lccc}
\hline Semen quality parameters & Clinical pregnancy & Biochemical pregnancy & Non-pregnancy \\
\hline Pre-processed TFMSC (million) & $92.21 \pm 70.21$ & $96.67 \pm 62.91$ & $84.71 \pm 60.26$ \\
Pre-processed N-TFMSC (million) & $135.53 \pm 82.91$ & $150.99 \pm 97.64$ & $127.16 \pm 83.53$ \\
Processed TFMSC (million) & $36.25 \pm 22.74$ & $40.28 \pm 23.93$ & $33.85 \pm 21.68$ \\
Processed N-TFMSC (million) & $4.40 \pm 3.15$ & $6.37 \pm 4.27^{\star}$ & $4.48 \pm 3.60$ \\
\hline
\end{tabular}

Values are mean $\pm \mathrm{SD}$; One-way ANOVA was used in this analysis. ${ }^{*} \mathrm{P}<0.05$, compared with clinical pregnancy or non-pregnancy. AlH, artificial insemination with the husband's semen; TFMSC, total forward motile sperm count; N-TFMSC, total non-forward and non-motile sperm count.

Table 3 The parameters of patients in processed N-TFMSC $\leq 5.0$ million and $>5.0$ million

\begin{tabular}{|c|c|c|}
\hline N-TFMSC (million) & $\leq 5.0$ & $>5.0$ \\
\hline Female age (year) & $30.25 \pm 3.83$ & $30.49 \pm 3.85$ \\
\hline Male age (year) & $31.68 \pm 4.46$ & $32.27 \pm 4.66^{\star}$ \\
\hline infertility duration (year) & $2.83 \pm 1.86$ & $2.93 \pm 2.09$ \\
\hline Ovulation induction cycle (\%) & $46.69(367 / 786)$ & $56.74(202 / 786)^{\star \star}$ \\
\hline Female BMI & $21.76+3.32$ & $22.33 \pm 3.51^{\star \star}$ \\
\hline Cycle count & $1.84 \pm 0.91$ & $1.88 \pm 0.91$ \\
\hline Morphology (\%) & $4.08 \pm 2.01$ & $4.74 \pm 2.43^{\star \star}$ \\
\hline Processed TFMSC (million) & $26.85 \pm 16.68$ & $50.59 \pm 23.04^{\star \star}$ \\
\hline Processed N-TFMSC (million) & $2.70 \pm 1.15$ & $8.47 \pm 3.84^{\star \star}$ \\
\hline
\end{tabular}

Values are mean $\pm \mathrm{SD}$; an independent sample $T$-test was used in this analysis. ${ }^{*} \mathrm{P}<0.05$ or ${ }^{* *} \mathrm{P}<0.01$, compared with $\mathrm{N}$-TFMSC $\leq 5.0$ million groups. N-TFMSC, total non-forward and non-motile sperm count; BMI, body mass index; TFMSC, total forward motile sperm count.

data in multiple groups were displayed as crosstabs and were compared with the $\chi^{2}$ test. The significance value of Pearson $\chi^{2}$ was $0.045(<0.05)$; the contingency coefficient was only 0.073 (in Table 4). That was presumed to be a weak correlation between the processed N-TFMSC and the outcome of AIH.

\section{Discussion}

Semen processing is the product of aided reproductive technology (ART). Unlike natural fertilization, semen suspension is optimized before AIH, removing the nonsperm components and abnormal sperms $(7,8)$. It has generally been presumed the pregnancy rate is associated with female age, the infertility duration, infertility type, the insemination cycle, the dominant follicles, and semen quality $(9,10)$. The processed semen suspension was equipped with hyperactivated motility (HAM) and fertilization to look forward to an improved AIH pregnancy rate. Many reports showed that the processed total motile sperm count (PTMS) could represent semen's quality more than other semen parameters $(3,5)$. Badawy et al. (11) suggested that PTMS should not be less than 5 million. With the increase of PTMS, the pregnancy rate of AIH is also increased. When it reached 10 million, a satisfactory pregnancy rate can be obtained (12). Here, we care more 
Table 4 The influence of processed N-TFMSC on the outcome of AIH

\begin{tabular}{|c|c|c|c|}
\hline The processed N-TFMSC (million) & Clinical pregnancy & Biochemical pregnancy & Non-pregnancy \\
\hline Count & 100 & 7 & 679 \\
\hline The percent of the group & $12.72 \%$ & $0.90 \%$ & $86.39 \%$ \\
\hline \multicolumn{4}{|l|}{$>5.0$} \\
\hline The percent of the group & $12.08 \%$ & $2.72 \%$ & $85.11 \%$ \\
\hline
\end{tabular}

The $\chi^{2}$ test analyzes the crosstabs among the multiple groups; $0 \%$ of the table's expected count is less than 5 ; the minimum expected count is 5.30. N-TFMSC, total non-forward and non-motile sperm count; AlH, artificial insemination with the husband's semen.

about the forward motile sperm for their HAM and fertilization ability. Therefore, TFMSC and N-TFMSC are used to be the semen quality parameters on the day of AIH. Even a perfectly processed semen suspension cannot completely remove the non-forward and non-motile sperm. We hypothesized that there might be some effects on AIH, as the non-forward and non-motile sperm is also delivered directly into the female uterine cavity.

In the study, we divided the pregnancy outcome into three parts rather than two parts: the clinical pregnancy, biochemical pregnancy, and non-pregnancy. It is interesting to note that the statistically significant difference is only found on processed N-TFMSC. The mean processed N-TFMSC in the biochemical pregnancy group was significantly higher than the other two groups. It reminded us that we should pay more attention to process N-TFMSC. Then the study was divided into two groups according to processed N-TFMSC. The significant difference we observed between them tend to be more male parameters, including male age, morphology in previously semen examination, and all the semen quality parameters on the day of AIH. The older the male, accompanied by more processed N-TFMSC. Whereas the highest of normal morphology does not mean lower processed N-TFMSC, it may be due to the previously semen examination's morphology. Also, the highest of processed N-TFMSC was often accompanied by higher processed TFMSC.

A statistical increase in biochemical pregnancy rate was observed when the processed N-TFMSC was $>5.0$ million. Biochemical pregnancy due primarily to the embryo implantation process has begun but has not been completed; its mechanism is still unclear. The zygote begins to bed on day 6-7 after fertilization; it experiences position, adhesion, and implantation. Any problem with the step may lead to the final failure of implantation. Embryo qualities affect the pregnancy outcome through its ability to implant and its development potential $(13,14)$. Semen is the participant in ensuring the quality of the embryo. Sometimes we observed that more processed N-TFMSC happened to the same patient in different AIH cycles regardless of any processing measure. Does that mean the semen of the patient itself has an unknown defect? Their sperm can escape apoptosis and cannot be easily eliminated by conventional semen processing. And the remaining defective sperms may release reactive oxygen species (ROS) or toxins, which may be harmful to the forward motile sperm. As we know, an excess of ROS causes oxidative stress and therefore causes DNA damage in the nucleus and mitochondria (15). However, with the higher processed TFMSC in group 2 (processed N-TFMSC $>5.0$ million), excessive motility semen is injected into the female reproductive tract, which may increase the chances of polyspermic fertilization (16).

The maternal environment is another crucial factor in a biochemical pregnancy. Endometrium receptivity, endocrine factors, infection, immune factors, psychosocial factors, etc. (17). We suspected that non-forward and nonmotile sperm remained in the uterine cavity and adhered to the endometrium, which may change the endometrium morphology and the expression of its related factors. It may affect the adhesion space between pinopodes and embryos. Factors involved in endometrial receptivity, including the expression of integrin, leukemia, inhibitory factor, interleukin-1, etc., may also change as the stress of the nonforward and non-motile sperms.

Implantation failure is presumed to occur due to impairment of embryo developmental potential and/or impairment of endometrial receptivity and the embryouterine dialogue $(18,19)$. Although non-forward and non- 
motile sperms do not compete for fertilization, they do deliver directly into the uterine cavity together with the forward motile sperm and result in adverse effects on implantation. As reported above, the biochemical pregnancy rate was $2.72 \%$ when processed N-TFMSC was $>5.0$ million $(\mathrm{P}<0.05)$. The contingency coefficient was 0.073 ; there was a weak correlation between the N-TFMSC and AIH outcome. This was a retrospective study with a limited sample size, especially since the number of biochemical pregnancies was only 17, which may lead to bias; further study is needed to confirm our conclusion.

In summary, the processed N-TFMSC may be one of the independent factors on AIH's outcome; it should be given equal attention the same as the processed TFMSC. We should control the processed N-TFMSC at least $\leq 5$ million; sometimes, the upstream method is necessary to lessen the processed N-TFMSC after the density gradient centrifugation of semen processing.

\section{Acknowledgments}

Funding: None.

\section{Footnote}

Reporting Checklist: The authors have completed the MDAR reporting checklist. Available at http://dx.doi.org/10.21037/ tau-20-1385

Data Sharing Statement: Available at http://dx.doi. org/10.21037/tau-20-1385

Conflicts of Interest: All authors have completed the ICMJE uniform disclosure form (available at http://dx.doi. org/10.21037/tau-20-1385). The authors have no conflicts of interest to declare.

Ethical Statement: The authors are accountable for all aspects of the work in ensuring that questions related to the accuracy or integrity of any part of the work are appropriately investigated and resolved. The study was approved by The Affiliated Hospital (Group) of Putian University (NO.[2015]229). All patients in the study signed informed consent. All procedures performed in this study involving human participants were in accordance with the Declaration of Helsinki (as revised in 2013).

Open Access Statement: This is an Open Access article distributed in accordance with the Creative Commons Attribution-NonCommercial-NoDerivs 4.0 International License (CC BY-NC-ND 4.0), which permits the noncommercial replication and distribution of the article with the strict proviso that no changes or edits are made and the original work is properly cited (including links to both the formal publication through the relevant DOI and the license). See: https://creativecommons.org/licenses/by-nc-nd/4.0/.

\section{References}

1. Kandavel V, Cheong Y. Does intra-uterine insemination have a place in modern ART practice? Best Pract Res Clin Obstet Gynaecol 2018;53:3-10.

2. Cohlen B, Bijkerk A, Van der Poel S, et al. IUI: review and systematic assessment of the evidence that supports global recommendations. Hum Reprod Update 2018;24:300-19.

3. Ombelet $W$, Dhont $N$, Thijssen A, et al. Semen quality and prediction of IUI success in male subfertility: a systematic review. Reprod Biomed Online 2014;28:300-9.

4. Cabry-Goubet R, Scheffler F, Belhadri-Mansouri N, et al. Effect of Gonadotropin Types and Indications on Homologous Intrauterine Insemination Success: A Study from 1251 Cycles and a Review of the Literature. Biomed Res Int 2017;2017:3512784.

5. Ombelet W, Deblaere K, Bosmans E, et al. Semen quality and intrauterine insemination. Reprod Biomed Online 2003;7:485-92.

6. Dong F, Sun Y, Su Y, et al. Relationship between processed total motile sperm count of husband or donor semen and pregnancy outcome following intrauterine insemination. Syst Biol Reprod Med 2011;57:251-5.

7. Sakkas D, Ramalingam M, Garrido N, et al. Sperm selection in natural conception: what can we learn from Mother Nature to improve assisted reproduction outcomes? Hum Reprod Update 2015;21:711-26.

8. Rappa KL, Rodriguez HF, Hakkarainen GC, et al. Sperm processing for advanced reproductive technologies: Where are we today? Biotechnol Adv 2016;34:578-87.

9. Osaikhuwuomwan J, Osemwenkha A, Iribhogbe O, et al. The effect of female age on the outcome of intrauterine insemination treatment in a public hospital-assisted reproduction technology unit. Niger J Clin Pract 2018;21:988-92.

10. Ahinko-Hakamaa K, Huhtala H, Tinkanen H. Success in intrauterine insemination: the role of etiology. Acta Obstet Gynecol Scand 2007;86:855-60.

11. Badawy A, Elnashar A, Eltotongy M. Effect of sperm 
morphology and number on success of intrauterine insemination. Fertil Steril 2009;91:777-81.

12. Miller DC, Hollenbeck BK, Smith GD, et al. Processed total motile sperm count correlates with pregnancy outcome after intrauterine insemination. Urology 2002;60:497-501.

13. Liu HC, Jones GS, Jones HW, Jr., et al. Mechanisms and factors of early pregnancy wastage in in vitro fertilizationembryo transfer patients. Fertil Steril 1988;50:95-101.

14. Troncoso C, Bosch E, Rubio C, et al. The origin of biochemical pregnancies: lessons learned from preimplantation genetic diagnosis. Fertil Steril 2003;79:449-50.

15. Bui AD, Sharma R, Henkel R, et al. Reactive oxygen species impact on sperm DNA and its role in male

Cite this article as: $\mathrm{Liu} \mathrm{L}, \mathrm{Wu} \mathrm{B}, \mathrm{Li} \mathrm{Z}$, Lin $\mathrm{H}$. The influence of processed total non-forward and non-motile sperm count on the outcome of artificial insemination with the husband's semen. Transl Androl Urol 2020;9(6):2730-2736. doi: 10.21037/ tau-20-1385 infertility. Andrologia 2018;50:e13012.

16. Wang WH, Day BN, Wu GM. How does polyspermy happen in mammalian oocytes? Microsc Res Tech 2003;61:335-41.

17. Annan JJ, Gudi A, Bhide P, et al. Biochemical pregnancy during assisted conception: a little bit pregnant. J Clin Med Res 2013;5:269-74.

18. Massimiani M, Lacconi V, La Civita F, et al. Molecular Signaling Regulating Endometrium-Blastocyst Crosstalk. Int J Mol Sci 2019;21.

19. Diedrich K, Fauser BC, Devroey P, et al. The role of the endometrium and embryo in human implantation. Hum Reprod Update 2007;13:365-77.

(English Language Editor: J. Chapnick) 